\title{
DISFONIAS: RELAÇÃO S/Z E TIPOS DE VOZ
}

\section{Dysphonias: $S / Z$ ratio and types of voice}

\author{
Carla Aparecida Cielo (1), Giseane Conterno (2), \\ Cláudia Dutra de Moraes Carvalho ${ }^{(3)}$, Leila Susana Finger ${ }^{(4)}$
}

\begin{abstract}
RESUMO
Objetivo: verificar o resultado da relação s/z e do tipo de voz em pacientes com diagnóstico de disfonias orgânico-funcionais (DOF) e disfonias funcionais por uso incorreto da voz (DFUIV), bem como a ocorrência das diferentes patologias dentro das DOF. Métodos: 70 indivíduos, de ambos os sexos, entre cinco e 65 anos de idade, atendidos numa clínica-escola, cadastrados em Banco de Dados, entre 1998 e 2006, com DOF e DFUIV, ambos classificados em três subgrupos: casos em que a relação s/z indicava hipercontração, normalidade, e falta de coaptação das pregas vocais durante a fonação. Os tipos de voz foram classificados conforme a ocorrência em: sem alteração; ruidosa; ruidosa, comprimida e/ou apresentando alteração de $f_{0} h$; ruidosa e/ou apresentando alteração de $f_{0} h$; e comprimida. Resultados: ocorrência significativa de DOF e de DOF com nódulos vocais; nas DOF e DFUIV, ocorrência significante de relação s/z normal com tempos isolados de /s/ e /z/ abaixo do normal e voz ruidosa. Conclusões: $O$ resultado da relação s/z foi estatisticamente significativo a favor da faixa de normalidade estabelecida, tanto nos pacientes com diagnóstico de DOF, quanto naqueles com DFUIV, sendo que, em ambos os grupos de pacientes, a voz ruidosa foi a mais freqüente. Dentro das DOF, os nódulos vocais foram significativamente mais freqüentes do que as demais patologias.
\end{abstract}

DESCRITORES: Voz; Fonação; Distúrbios da Voz; Avaliação em Saúde

\section{INTRODUÇÃO}

As perturbações do fluxo expiratório podem ser conseqüências do uso inadequado da musculatura respiratória, que ocasiona um conflito entre a pressão subglótica e a resistência glótica. A alteração da vibração das pregas vocais pode ser produzida pela presença de lesões estruturais, pela existência de hipertonia da musculatura laríngea ou pela deficiência da coaptação glótica ${ }^{1,2}$. Em estudos realizados observou-se em população de pacientes com disfonia funcional ${ }^{3-6}$ e orgânico-funcional características evidentes de hiperfunção glótica ${ }^{5,7}$.

(1) Fonoaudióloga; Professora Adjunta do Departamento de Fonoaudiologia da Universidade Federal de Santa Maria; Doutora em Linguagem Aplicada pela Pontifícia Universidade Católica do Rio Grande do Sul.

(2) Fonoaudióloga; Mestranda em Distúrbios da Comunicação Humana pela Universidade Federal de Santa Maria.

(3) Fonoaudióloga; Clínica Cláudia Carvalho.

(4) Fonoaudióloga; Bolsista Coordenação de Aperfeiçoamento de Pessoal de Nível Superior; Mestranda em Distúrbios da Comunicação Humana pela Universidade Federal de Santa Maria.
A avaliação perceptivo-auditiva da voz é uma forma subjetiva de identificar alterações na qualidade vocal, baseando-se no conhecimento prévio do examinador e na sua capacidade de discriminar as diferentes características da voz normal e patológica $^{8}$. Um dos aspectos vocais perceptivo-auditivo comumente avaliado da qualidade vocal é o tipo de voz, que é diretamente relacionado à seleção dos ajustes motores empregados, tanto em nível de prega vocal quanto em nível de ressonância, permitindo verificar dados sobre a qualidade vocal, o pitch e a loudness ${ }^{9,10}$.

Outro método clássico e habitual utilizado no auxílio do diagnóstico das disfonias é a medida do Tempo Máximo de Fonação (TMF), que utiliza, dentre outros, os fonemas fricativos /s/ e /z/ 9,11. Tais TMF são obtidos e cronometrados durante a fonação sustentada em uma única expiração e fornecem dados sobre a dinâmica de fonação, sendo bastante fidedignos em avaliação de eficiência glótica ${ }^{9,12}$.

Segundo a literatura ${ }^{12,13}$, a relação s/z permite verificar a ocorrência do componente de hipercontração muscular e do componente de falta de coaptação das pregas vocais em pacientes com diagnóstico de disfonia funcional por uso incorreto da 
voz (DFUIV) e disfonia orgânico-funcional (DOF), por isso a inter-relação entre as disfonias supracitadas, os tipos de voz encontrados, e a relação s/z é o foco principal do presente estudo, cujo objetivo foi verificar o resultado da relação s/z e do tipo de voz em pacientes com diagnóstico de disfonias orgânico-funcionais (DOF) e disfonias funcionais por uso incorreto da voz (DFUIV), bem como a ocorrência das diferentes patologias dentro das DOF.

\section{MÉTODOS}

Trata-se de um estudo transversal, exploratório, não experimental, quanti-qualitativo de dados obtidos do Banco de Dados dos pacientes com queixas de voz atendidos no Serviço de Atendimento Fonoaudiológico da clínica-escola da Universidade Federal de Santa Maria/RS.

A população alvo constituiu-se de 112 sujeitos atendidos na clínica no período de janeiro de 1998 a agosto de 2006.

Os pacientes atendidos nesta clínica assinam previamente um termo de consentimento livre e esclarecido, no qual autorizam o uso dos dados de avaliação e tratamento, em pesquisas científicas, desde que preservadas suas identidades. Os dados do estudo, quanto aos TMF, patologias, e tipos de voz, foram retirados do Banco de Dados dos pacientes com queixas de voz e, como esta pesquisa não possui caráter experimental, não houve necessidade de assinatura de novo termo de consentimento livre e esclarecido. Os critérios de inclusão para a pesquisa foram diagnóstico otorrinolaringológico e fonoaudiológico prévio de alterações orgânico-funcionais e funcionais por uso incorreto da voz, o que resultou um total de 70 indivíduos, de ambos os sexos, com idades variando entre cinco e 65 anos, com média de 27,67 anos, sendo 48 sujeitos com disfonia orgânico-funcional (DOF) e 22 com disfonia funcional por uso incorreto da voz (DFUIV).

Os TMF foram cronometrados por diferentes avaliadores, os sujeitos eram orientados a, em pé, inspirar pelo nariz e emitir os fonemas /s/ e /z/ em tom, intensidade e velocidade habituais, sustentando-os durante o tempo máximo possível durante a mesma expiração ${ }^{9}$, por três vezes, sendo escoIhida a maior sustentação.

Os casos selecionados foram classificados em três grupos: os casos em que a relação $s / z$ indicava normalidade, aqueles em que a relação $s / z$ indicava hipercontração glótica, e os casos em que a relação $\mathrm{s} / \mathrm{z}$ indicava falta de coaptação das pregas vocais durante a fonação.

Para os TMF /s/ e /z/ dentro dos padrões de normalidade, em adultos (a partir de doze anos ${ }^{13}$ ), foi considerada uma média do valor mínimo e do máximo dentre os estudos já realizados sobre TMF. O valor médio mínimo foi 14,64 s e o valor médio máximo $26,58 \mathrm{~s}^{1,9}$.

Para o padrão de normalidade do TMF em crianças (até doze anos de idade incompletos ${ }^{14}$ ), foi considerada uma média do valor mínimo e do máximo, dentre os estudos já realizados sobre TMF em crianças, por faixa etária: para 5 anos, de 5 a 9,36 s e para 6 anos, de 6 a 14,2s 9,13,15-18. Para a faixa etária de 7 a 11 anos, como não foram encontrados intervalos mínimos e máximos de TMF, foi considerado o critério de médias para cada idade: para 7 anos, 13,23s; para 8 anos, 13,57s; para 9 anos, 13,90s; para 10 anos, 14,23s; e para 11 anos, $14,57 s^{9.13}$.

Os TMF /z/ abaixo dos intervalos de normalidade foram considerados como sugestivos de falta de coaptação das pregas vocais; e os que se situaram acima dos intervalos de normalidade foram considerados sugestivos de hipercontração glótica durante a fonação.

Os TMF /s/ abaixo dos intervalos de normalidade foram considerados como sugestivos de falta de controle expiratório à fonação, e os situados acima da normalidade foram sugestivos de controle aéreo melhor que o esperado ${ }^{9,13}$.

Uma relação s/z em torno de 1 é considerado o padrão de normalidade ${ }^{9,13,19-24}$. Porém, neste estudo, foram considerados, para padrões de normalidade da relação $\mathrm{s} / \mathrm{z}$, os valores compreendidos entre $0,82^{9,16,21,25,26}$ a $1,23^{1,9,16,19-21,25,26}$, sendo que tais limites foram obtidos a partir da realização da média dos valores considerados normais, propostos na literatura consultada.

Para a relação $s / z$, os resultados abaixo dos intervalos de normalidade foram interpretados como indicativos de hipercontração glótica durante a fonação; e acima, foram considerados como indicativos de falta de coaptação glótica ${ }^{9,21}$.

Como os resultados da relação $\mathrm{s} / \mathrm{z}$ não mostram diferença marcante entre os sexos ${ }^{16,24}$, e com o aumento de idade ${ }^{16}$, conforme a literatura, o padrão de normalidade foi considerado o mesmo para ambos os sexos e para adultos e crianças.

Para a tabulação dos dados, considerou-se o número de sujeitos, incluindo-se adultos e crianças, de ambos os sexos, com resultado de TMF ou de relação s/z dentro ou fora da normalidade, conforme o esperado para cada categoria.

Os tipos de voz, qualificados através de análise perceptivo-auditiva, foram classificados em diferentes categorias, conforme a sua ocorrência isolada ou associada a outro tipo, segundo opção dos pesquisadores do presente estudo, em: sem alteração; ruidosa; ruidosa, comprimida e/ou apresentando alteração de freqüência fundamental $\left(f_{0} h\right)$; ruidosa 
e/ou apresentando alteração de $\mathrm{f}_{0} \mathrm{~h}$; e comprimida. Foi considerada alteração de $f_{0} h$ a presença de tremor, monotonia, bitonalidade, virilização, agravamento e/ou diplofonia; e como ruído, a presença de rouquidão, aspereza e/ou soprosidade.

O projeto que dá base ética e metodológica ao Banco de Dados do Setor de Voz foi previamente aprovado pelo Comitê de Ética em Pesquisa da instituição de origem, sob o número 074/2005.

A análise estatística dos dados foi realizada por meio do teste de hipótese paramétrico bilateral para diferença entre duas proporções amostrais. Em todos os casos, adotou-se um nível de significância $(\alpha)$ de $5 \%$. O objetivo do teste foi determinar se a amostra foi significativa; foram analisadas todas as proporções de cada tabela, para cada uma das variáveis e verificados se eram significativamente iguais ou diferentes; também foram realizados cruzamentos entre todas as variáveis utilizadas e proporções em relação à amostra total. As interpretações que apresentaram significância encontram-se nas tabelas a seguir.

\section{RESULTADOS}

Na Tabela 1 (Porcentagem de casos de DFUIV e de DOF), observou-se significância estatística a favor da DOF $(p=0,0000)$.
Tabela 1 - Casos de DFUIV e de DOF

\begin{tabular}{lccc}
\hline \multicolumn{4}{c}{ DFUIV x DOF } \\
\hline DFUIV & $\mathrm{N}$ & $\%$ & Valor de $\mathrm{p}$ \\
DOF & 22 & 31 & $\begin{array}{c}\text { DFUIV } \\
= \\
\mathrm{DOF}^{*} \\
\mathrm{p}=0,0000\end{array}$ \\
\hline
\end{tabular}

* Significância estatística

Teste de hipótese paramétrico bilateral para diferença entre duas proporções amostrais

Na Tabela 2 (TMF /z/ nas DFUIV e DOF), observou-se, dentro das DFUIV, significância estatística a favor da Normalidade $(p=0,0294)$ e a favor da Falta de coaptação glótica ( $p=0,0514)$, quando comparadas à Hipercontração glótica. Dentro das DOF, pôde-se observar significância estatística a favor da Falta de coaptação glótica $(p=0,0000)$, quando comparada à Normalidade; significância estatística a favor da Falta de coaptação glótica $(p=0,0000)$, quando comparada à Hipercontração glótica; e significância estatística a favor da Normalidade $(p=0,0201)$, quando comparada à Hipercontração glótica.

Na Tabela 3 (TMF /s/ nas DFUIV e DOF), observou-se, dentro das DFUIV, significância estatística a favor da Normalidade $(p=0,0185)$ e a favor da Falta de controle expiratório $(p=0,0048)$, quando

Tabela 2 - TMF /z/ nas DFUIV e DOF

\begin{tabular}{|c|c|c|c|c|}
\hline \multicolumn{5}{|c|}{ DFUIV e DOF / TMF /z/ } \\
\hline & & $\mathrm{N}$ & $\%$ & Valor de $p$ \\
\hline \multirow{4}{*}{ DFUIV } & Hipercontração glótica & 3 & 14 & Hipercontração glótica \\
\hline & Normalidade & 10 & 45 & $\begin{array}{c}= \\
\text { Normalidade* } \\
\mathrm{p}=0,0294\end{array}$ \\
\hline & Hipercontração glótica & 3 & 14 & $\begin{array}{c}\text { Hipercontração glótica } \\
=\end{array}$ \\
\hline & Falta de coaptação glótica & 9 & 41 & $\begin{array}{l}\text { Falta de coaptação glótica* } \\
\qquad p=0,0514\end{array}$ \\
\hline \multirow{6}{*}{ DOF } & Normalidade & 11 & 23 & Normalidade \\
\hline & Falta de coaptação glótica & 34 & 71 & $\begin{array}{l}= \\
\text { Falta de coaptação glótica* } \\
\qquad \begin{array}{l}\mathrm{p}=0,0000 \\
\end{array}\end{array}$ \\
\hline & Falta de coaptação glótica & 34 & 71 & Falta de coaptação glótica* \\
\hline & Hipercontração glótica & 3 & 6 & $\begin{array}{c}= \\
\text { Hipercontração glótica } \\
p=0,0000\end{array}$ \\
\hline & Normalidade & 11 & 23 & $\begin{array}{c}\text { Normalidade }^{*} \\
=\end{array}$ \\
\hline & Hipercontração glótica & 3 & 6 & $\begin{array}{l}\text { Hipercontração glótica } \\
\qquad p=0,0201\end{array}$ \\
\hline
\end{tabular}

* Significância estatística

Teste de hipótese paramétrico bilateral para diferença entre duas proporções amostrais 


\begin{tabular}{|c|c|c|c|c|}
\hline \multicolumn{5}{|c|}{ DFUIV e DOF / TMF /s/ } \\
\hline & & $\mathrm{n}$ & $\%$ & Valor de $\mathrm{p}$ \\
\hline \multirow{4}{*}{ DFUIV } & Normalidade & 9 & 41 & Normalidade $^{*}$ \\
\hline & Melhor controle expiratório & 2 & 9 & $\begin{array}{c}= \\
\text { Melhor controle expiratório } \\
p=0,0185\end{array}$ \\
\hline & Falta de controle expiratório & 11 & 50 & Falta de controle expiratório* \\
\hline & Melhor controle expiratório & 2 & 9 & $\begin{array}{l}\text { Melhor controle expiratório } \\
\qquad p=0,0048\end{array}$ \\
\hline \multirow{6}{*}{ DOF } & Normalidade & 15 & 31 & $\begin{array}{c}\text { Normalidade } \\
=\end{array}$ \\
\hline & Falta de controle expiratório & 29 & 61 & $\begin{array}{l}\text { Falta de controle expiratório* } \\
\qquad p=0,0040\end{array}$ \\
\hline & Normalidade & 15 & 31 & Normalidade ${ }^{*}$ \\
\hline & Melhor controle expiratório & 4 & 8 & $\begin{array}{c}= \\
\text { Melhor controle expiratório } \\
p=0,0055\end{array}$ \\
\hline & Falta de controle expiratório & 29 & 61 & $\begin{array}{c}\text { Falta de controle expiratório* } \\
=\end{array}$ \\
\hline & Melhor controle expiratório & 4 & 8 & $\begin{array}{l}\text { Melhor controle expiratório } \\
\qquad \mathrm{p}=0,0000\end{array}$ \\
\hline
\end{tabular}

* Significância estatística

Teste de hipótese paramétrico bilateral para diferença entre duas proporções amostrais

comparadas ao Melhor controle expiratório. Dentro das DOF, pôde-se observar significância estatística a favor da Falta de controle expiratório $(p=0,0040)$, quando comparada à Normalidade; significância estatística a favor da Normalidade $(p=0,0055)$, quando comparada ao Melhor controle expiratório; e significância estatística a favor da Falta de controle expiratório $(p=0,0000)$, quando comparado ao Melhor controle expiratório.

$\mathrm{Na}$ Tabela 4 (Relação s/z nas DFUIV e DOF), observou-se: significância estatística a favor da Normalidade $(p=0,0258)$, quando comparada à Falta de coaptação glótica; significância estatística a favor da Normalidade $(p=0,0000)$, quando comparada à Hipercontração glótica; e significância estatística a favor da Falta de coaptação glótica $(p=0,0000)$, quando comparada à Hipercontração glótica. Levando-se em consideração somente as DFUIV, pôde-se observar: significância estatística a favor da Normalidade $(p=0,0457)$, quando comparada à Falta de coaptação glótica; significância estatística a favor da Normalidade $(p=0,0002)$, quando comparada à Hipercontração glótica; e significância estatística a favor da Falta de coaptação glótica $(p=0,0261)$, quando comparada à Hipercontração glótica. Levando-se em consideração somente as DOF, pôde-se observar: significância estatística a favor da Normalidade $(p=0,0000)$, quando comparada à Hipercontração glótica; e significância estatística a favor da Falta de coaptação glótica $(p=0,0004)$, quando comparada à Hipercontração glótica.

Na Tabela 5 (Tipos de voz nas DFUIV e DOF), observou-se: significância estatística a favor do Com alteração $(p=0,0000)$, quando comparado ao Sem alteração; significância estatística a favor do Ruído (rouquidão, aspereza e/ou soprosidade) $(p=0,0000)$, quando comparado ao Ruído, compressão e /ou alteração de fOh; significância estatística a favor do Ruído (rouquidão, aspereza e/ou soprosidade) $(p=0,0000)$, quando comprado ao Compressão; e significância estatística a favor do Ruído (rouquidão, aspereza e/ou soprosidade) $(p=0,0000)$, quando comparado ao Ruído e/ou alteração de fOh. Levando-se em consideração somente as DFUIV, pôde-se observar: significância estatística a favor do Com alteração ( $p=0,0009)$, quando comparado ao Sem alteração; significância estatística a favor do Ruído (rouquidão, aspereza e/ou soprosidade) ( $p=0,0000)$, quando comparado à Sem alteração; significância estatística a favor do Ruído (rouquidão, aspereza e/ou soprosidade) ( $p=0,0165)$, quando comprado com Ruído, compressão e/ou alteração de fOh; significância estatística a favor do Ruído (rouquidão, aspereza e/ou soprosidade) ( $p=0,0021)$, quando comparado à Compressão; e significância estatística a favor do Ruído (rouquidão, aspereza e/ou soprosidade) 
Tabela 4 - Relação s/z nas DFUIV e DOF

\begin{tabular}{|c|c|c|c|c|}
\hline \multicolumn{5}{|c|}{ DFUIV e DOF / Relação s/z } \\
\hline & & $\mathrm{N}$ & $\%$ & Valor de $p$ \\
\hline \multirow{6}{*}{ DFUIV e DOF } & Normalidade & 39 & 56 & Normalidade* $^{*}$ \\
\hline & Falta de coaptação glótica & 26 & 37 & $\begin{array}{l}\text { Falta de coaptação glótica } \\
\qquad p=0,0258\end{array}$ \\
\hline & Normalidade & 39 & 56 & Normalidade* $^{\star}$ \\
\hline & Hipercontração glótica & 5 & 7 & $\begin{array}{c}= \\
\text { Hipercontração glótica } \\
p=0,0000\end{array}$ \\
\hline & Falta de coaptação glótica & 26 & 37 & $\begin{array}{c}\text { Falta de coaptação glótica* } \\
=\end{array}$ \\
\hline & Hipercontração glótica & 5 & 7 & $\begin{array}{l}\text { Hipercontração glótica } \\
\text { p=0,0000 }\end{array}$ \\
\hline \multirow{6}{*}{ DFUIV } & Normalidade & 14 & 64 & Normalidade* \\
\hline & Falta de coaptação glótica & 7 & 32 & $\begin{array}{l}\text { Falta de coaptação glótica } \\
\qquad p=0,0457\end{array}$ \\
\hline & Normalidade & 14 & 64 & Normalidade $^{*}$ \\
\hline & Hipercontração glótica & 1 & 5 & $\begin{array}{c}= \\
\text { Hipercontração glótica } \\
p=0,0002\end{array}$ \\
\hline & Falta de coaptação glótica & 7 & 32 & $\begin{array}{c}\text { Falta de coaptação glótica* } \\
=\end{array}$ \\
\hline & Hipercontração glótica & 1 & 5 & $\begin{array}{l}\text { Hipercontração glótica } \\
\qquad p=0,0261\end{array}$ \\
\hline \multirow{4}{*}{ DOF } & Normalidade & 25 & 52 & $\begin{array}{c}\text { Normalidade }^{*} \\
=\end{array}$ \\
\hline & Hipercontração glótica & 4 & 8 & $\begin{array}{l}\text { Hipercontração glótica } \\
\qquad p=0,0000\end{array}$ \\
\hline & Falta de coaptação glótica & 19 & 40 & $\begin{array}{c}\text { Falta de coaptação glótica* } \\
=\end{array}$ \\
\hline & Hipercontração glótica & 4 & 8 & $\begin{array}{l}\text { Hipercontração glótica } \\
\qquad p=0,0004\end{array}$ \\
\hline
\end{tabular}

* Significância estatística

Teste de hipótese paramétrico bilateral para diferença entre duas proporções amostrais

( $p=0056$ ), quando comparado ao Ruído e/ou alteração de fOh. Levando-se em consideração somente as DOF, pôde-se observar: significância estatística a favor do Com alteração ( $p=0,0000)$, quando comparado ao Sem alteração; significância estatística a favor do Ruído (rouquidão, aspereza e/ou soprosidade) $(p=0,0000)$, quando comparado à Sem alteração; significância estatística a favor do Ruído (rouquidão, aspereza e/ou soprosidade) $(p=0,0000)$, quando comprado com Ruído, compressão e/ou alteração de fOh; significância estatística a favor do Ruído (rouquidão, aspereza e/ou soprosidade) $(p=0,0000)$, quando comparado à Compressão; e significância estatística a favor do Ruído (rouqui- dão, aspereza e/ou soprosidade) $(\mathrm{p}=0000)$, quando comparado ao Ruído e/ou alteração de foh.

$\mathrm{Na}$ Tabela 6 (Patologias na DOF), foi observada significância estatística a favor do Nódulo $(p=0,0000)$, quando comparado ao Cisto, ao Edema de prega vocal, à Assimetria de prega vocal, ao Pólipo e ao Edema de Reinke.

Na Tabela 7 (Significância do TMF /s/ , TMF /z/, relação s/z e tipos de voz entre as DFUIV e DOF), observou-se significância estatística em Sem alteração $(p=0,0049)$ a favor da DFUIV, significância estatística no Ruído $(p=0,0166)$ a favor da DOF, e significância estatística na Falta de coaptação glótica $(T M F / z /)(p=0,0194)$ a favor da DOF. 
Tabela 5 - Tipos de voz nas DFUIV e DOF



* Significância estatística

Teste de hipótese paramétrico bilateral para diferença entre duas proporções amostrais 
Tabela 6 - Patologias na DOF

\begin{tabular}{|c|c|c|c|}
\hline \multicolumn{4}{|c|}{ DOF/ Patologias } \\
\hline & $\mathrm{n}$ & $\%$ & Valor de $p$ \\
\hline Nódulo & 36 & 75 & $\begin{array}{c}\text { Nódulo* } \\
=\end{array}$ \\
\hline Cisto & 6 & 13 & $\begin{array}{c}\text { Cisto } \\
p=0,0000\end{array}$ \\
\hline Nódulo & 36 & 75 & $\begin{array}{c}\text { Nódulo* } \\
=\end{array}$ \\
\hline Edema de prega vocal & 2 & 4 & $\begin{array}{l}\text { Edema de prega vocal } \\
\qquad p=0,0000\end{array}$ \\
\hline Nódulo & 36 & 75 & $\begin{array}{c}\text { Nódulo* } \\
=\end{array}$ \\
\hline Assimetria de prega vocal & 1 & 2 & $\begin{array}{l}\text { Assimetria de prega vocal } \\
\qquad p=0,0000\end{array}$ \\
\hline Nódulo & 36 & 75 & $\begin{array}{l}\text { Nódulo* } \\
=\end{array}$ \\
\hline Pólipo & 2 & 4 & $\begin{array}{c}\text { Pólipo } \\
p=0,0000\end{array}$ \\
\hline Nódulo & 36 & 75 & $\begin{array}{c}\text { Nódulo* } \\
=\end{array}$ \\
\hline Edema de Reinke & 1 & 2 & $\begin{array}{l}\text { Edema de Reinke } \\
\qquad p=0,0000\end{array}$ \\
\hline
\end{tabular}

* Significância estatística

Teste de hipótese paramétrico bilateral para diferença entre duas proporções amostrais

Tabela 7 - Significância do TMF /s/, TMF /z/, relação s/z e tipos de voz entre as DFUIV e DOF

\begin{tabular}{lccccccc}
\hline \multicolumn{7}{c}{ DFUIV X DOF } \\
\hline & $\begin{array}{c}\text { Falta de } \\
\text { controle } \\
\text { expiratório }\end{array}$ & Normalidade & $\begin{array}{c}\text { Melhor } \\
\text { controle } \\
\text { expiratório }\end{array}$ & $\begin{array}{c}\text { Falta de } \\
\text { coaptação } \\
\text { glótica }\end{array}$ & $\begin{array}{c}\text { Hipercontra- } \\
\text { ção glótica }\end{array}$ & $\begin{array}{c}\text { Sem } \\
\text { alteração }\end{array}$ & Ruído \\
\hline TMF/s/ & $\mathrm{p}=0,4356$ & $\mathrm{p}=0,4156$ & $\mathrm{p}=0,8885$ & - & - & - & - \\
TMF/z/ & - & $\mathrm{p}=0,0663$ & - & $\mathrm{p}=0,0194^{*}$ & $\mathrm{p}=0,2695$ & - & - \\
Relação $\mathbf{s} / \mathbf{z}$ & - & $\mathrm{p}=0,3930$ & - & $\mathrm{p}=0,05231$ & $\mathrm{p}=0,6506$ & - & - \\
Tipos de Voz & - & - & - & - & - & $\mathrm{p}=0,0049^{* *}$ & $\mathrm{p}=0,0166^{*}$ \\
\hline
\end{tabular}

* Significância estatística a favor DOF, quando consideradas a linha e a coluna onde figura o *

* * Significância estatística a favor DFUIV, quando consideradas a linha e a coluna onde figuram os *

Teste de hipótese paramétrico bilateral para diferença entre duas proporções amostrais (DOF X DFUIV)

\section{DISCUSSÃO}

Nos resultados desta pesquisa, foi encontrado um percentual significativo de sujeitos apresentando DOF (Tabela 1), indo ao encontro da literatura que afirma que as mesmas são DFUIV com diagnóstico tardio devido à demora de solução do problema ou pelo desconhecimento da possibilidade de desenvolvimento de uma lesão ${ }^{27}$. Verificase, assim, que esses altos índices provavelmente são provenientes de comportamentos vocais inadequados por tempo suficiente para ocasionar lesões nas pregas vocais. Tais afirmações são pertinentes ao se analisar um estudo ${ }^{28} \mathrm{com} 772$ professores, no qual foi observado que a patologia mais comum era a DFUIV, aparecendo em $87 \%$ dos casos, com $18 \%$ dos mesmos tendo evoluído para uma DOF.

Constatou-se, neste trabalho, que os TMF /s/ e /z/ na DOF apresentam-se estatisticamente reduzidos na maior parte da amostra (Tabelas 2 e 3 ), porém a relação $\mathrm{s} / \mathrm{z}$, em $52 \%$ da amostra está dentro do padrão de normalidade (Tabela 4). Os dados desta relação mostram apenas que ambas as fricativas apresentam TMF equilibrados entre si, mas os TMF das mesmas, se analisados isoladamente, sugerem que a maior parte dos sujeitos apresentaram falta de coordenação de controle expiratório e/ou falta de coaptação glótica. 
Estes resultados vão ao encontro de um estudo ${ }^{24}$ em que, para se utilizar a relação $\mathrm{s} / \mathrm{z} \mathrm{com}$ efeito diagnóstico, deve-se examinar o TMF /s/ e /z/ isoladamente, o fluxo de ar, bem como a intensidade com que foram produzidos, para então determinar se a base teórica da relação s/z é de fato válida.

Há também uma controvérsia na literatura apontando que a produção do fonema /s/ não é considerada fidedigna para se verificar o controle do sopro por suporte respiratório exclusivamente, devido ao bloqueio linguodental da articulação do fonema que pode atuar reduzindo a passagem do ar expiratório e prolongando o TMF. Desta forma, foi proposta a relação entre os tempos de emissão de /e/ áfono e de /e/ sonoro, já que a medida isolada da vogal áfona /e/ é um importante indicador clínico de como o paciente controla a saída progressiva do ar, por meio exclusivamente de seu suporte respiratório ${ }^{21}$.

No entanto, do grupo DOF, $40 \%$ dos sujeitos apresentaram a relação $\mathrm{s} / \mathrm{z}$ indicativa de falta de coaptação glótica (Tabela 4), o que pode estar relacionado ao fato do ruído (rouquidão, soprosidade e aspereza) ter sido o tipo de voz estatisticamente significativo predominante nesse grupo (Tabela 5), pois, na voz soprosa, ouve-se a voz acompanhada de ar não-sonorizado pelas pregas vocais, com a presença audível de fluxo de ar contínuo através da glote ${ }^{29}$, sendo que, ao exame otorrinolaringológico, tem-se coaptação deficiente das pregas vocais ${ }^{9,30}$ e freqüente presença de fenda glótica ${ }^{21}$; na voz áspera, o esforço do falante é evidente, sendo que esse tipo de voz é freqüentemente acompanhado de edema e rigidez de mucosa, na presença ou ausência de fenda glótica ${ }^{21}$; já a qualidade vocal rouca é, na verdade, uma qualidade mista, que contém elementos de soprosidade e aspereza, e geralmente está relacionada a lesões orgânicas e quadros orgânico-funcionais ${ }^{11}$.

Os nódulos vocais são a típica lesão orgânicofuncional, ou seja, decorrentes de usos vocais incorretos ${ }^{13,21,31}$. A presença desse tipo de lesão pode impedir que a glote se feche completamente durante a fonação, ocasionando fenda glótica e escape de ar fonatório ${ }^{32}$. Em determinado estudo ${ }^{1}$, constatou-se que os sujeitos com patologia nodular apresentavam tipo de voz áspera em $65 \%$ dos casos, soprosa em $54,1 \%$, e voz forçada em $37,5 \%$. Noutro trabalho ${ }^{32}$, quando foram avaliados pacientes portadores de nódulos vocais, a queixa principal de $96 \%$ dos indivíduos foi a rouquidão. Estas pesquisas mostram que, na presença da patologia nodular, a alteração vocal é evidente, sendo a rouquidão e soprosidade as perturbações mais encontradas, o que foi característico também nos achados do presente trabalho, quando se verificou significativo freqüência de nódulos vocais no grupo de DOF (Tabela 6).

A presença de alteração vocal no grupo de DOF foi estatisticamente significativo, sendo que $98 \%$ do grupo apresentaram alteração vocal (Tabela 5). Esses dados sugerem que a presença de patologia em nível de prega vocal geralmente ocasiona alteração da qualidade perceptivo-auditiva da voz.

Dentro do grupo de DFUIV, verificou-se que $50 \%$ dos sujeitos apresentaram TMF /s/ considerado reduzido (Tabela 3 ), podendo indicar incoordenação pneumo-fono-articulatória, já que o TMF /s/ é uma medida do controle expiratório ${ }^{26}$.

$\mathrm{Na}$ emissão sustentada do fonema /z/, que acrescenta à tarefa do controle do sopro expiratório o componente laríngeo ${ }^{26}, 45 \%$ dos sujeitos obtiveram resultado considerado normal (Tabela 2 ), sendo indicativo de ausência de patologia em nível de borda de prega vocal, já que os casos de DFUIV não apresentam lesões orgânicas ao exame laringoscópio $23,27,33$; porém, $41 \%$ dos sujeitos apresentaram TMF /z/ reduzido (Tabela 2), representando uma manifestação de atividade excessiva dos músculos laríngeos intrínsecos e extrínsecos durante a fonação ${ }^{34}$ e/ou uma incoordenação do esforço respiratório com o fechamento glótico ${ }^{13}$.

Na relação s/z do grupo de DFUIV, encontrou-se $63 \%$ dentro dos padrões de normalidade (Tabela 4), porém, na análise perceptivo-auditiva da voz, constatou-se que $77 \%$ dos sujeitos apresentavam algum tipo de alteração vocal (Tabela 5). Tais dados são compatíveis com a literatura, pois os casos de DFUIV, apesar de não apresentarem lesão orgânica ao exame laringológico, podem apresentar alteração em nível de ressonância, respiração, pitch, loudness, modulação e /ou gama tonal ${ }^{35}$.

As alterações de voz encontradas nas DFUIV ainda podem ser explicadas pela presença de rigidez do sistema fonatório, incluindo aumento da adução glótica e hipercinesia do trato vocal, o que pode causar aspereza, presença de fendas glóticas com escape de ar, alteração de coordenação pneumofono-articulatória e projeção deficiente, fatores que podem causar a rouquidão e a soprosidade. Além disso, o uso incorreto da voz pode causar alterações de freqüência ${ }^{13,21}$.

Tanto no grupo de DFUIV quanto no de DOF, houve significância dos casos com relação s/z indicando normalidade (Tabela 4), entretanto, a relação $\mathrm{s} / \mathrm{z}$ nesses dois grupos apresentou resultados indicativos de falta de coaptação glótica durante tempos de emissão significativamente maiores do que os resultados de hipercontração glótica durante a fonação. O fluxo de ar excessivo através da glote é geralmente um reflexo do fechamento glótico inadequado, o que ocorre na maioria das patologias 
da DOF (nódulos, pólipos, cistos, entre outras) ${ }^{13}$. A fadiga vocal, característica da DFUIV ${ }^{36}$, também pode gerar a presença de fenda glótica anterior, fechamento incompleto e fenda fusiforme, além de alterações no padrão vibratório das pregas vocais e de aumento de edema em nível de prega vocal ${ }^{21,37}$.

Os resultados do TMF /s/ para cada um dos grupos apontaram o mesmo: a falta de controle expiratório foi significativamente maior (Tabela 3 ), sugerindo que a incoordenação pneumo-fonoarticulatória pela falta de adequado suporte aéreo torna-se mais um fator de sobrecarga laríngea à fonação. A presença de incoordenação pneumofono-articulatória pode ocorrer também pelo predomínio de ruído (Tabela 5) e de nódulos vocais (Tabela 6), pois o trato vocal infraglótico funciona como fonte de força para a vocalização, podendose observar que alterações na estrutura ou função muscular resultam em um esforço compensatório da musculatura da laringe, o que pode provocar redução da função, fadiga vocal, dor e formação de nódulos vocais ${ }^{21,38}$.

A presença de voz ruidosa foi significativamente maior tanto no grupo de DFUIV quanto no de DOF (Tabela 5), porém foi constatada significância estatística a favor do ruído, quando comparado às vozes sem alteração, somente no grupo de DOF (Tabela 7). Assim, acredita-se que o uso incorreto da voz pode se manifestar de outras formas além da alteração do tipo de voz, o que é convergente com a literatura que identifica a DFUIV também por sensações negativas durante a fonação, como fadiga, sensação de volume faríngeo e laríngeo, dor na área laríngea ou torácica, secura na boca ao falar, constrição no pescoço, baixa resistência vocal, e incoordenação pneumo-fono-articulatória ${ }^{37}$.

O grupo de DOF apresentou tipo de voz ruidosa significativamente maior do que o grupo de DFUIV (Tabela 7), sugerindo que, quando a patologia já está instalada, a tendência à soprosidade (dificuldade de fechamento glótico), à rouquidão (aperiodicidade vibratória), e à aspereza (rigidez da mucosa ou do sistema fonatório) são maiores devido às características biomecânicas alteradas das pregas vocais em presença da lesão ${ }^{13}$. Nos pacientes com patologia laríngea, constataram-se também maiores índices de perturbação ciclo-a-ciclo da freqüência (jitter), e da intensidade (shimmer), e aumento de ruído glótico, sendo o shimmer mais relacionado à redução de resistência glótica e, consequentemente, à soprosidade e o jitter à rouquidão e aspereza vocais ${ }^{13,21,39,41}$.

Tais afirmações estão de acordo com os resultados de um estudo, no qual foram avaliados 70 pacientes portadores de nódulos vocais, no qual a queixa principal encontrada em $96,4 \%$ foi a rouquidão ${ }^{32}$. Verificou-se, também, por meio de pesquisa em que foram avaliadas 107 amostras de voz de pacientes com diagnóstico de edema de Reinke, que o tipo de voz resultante dessa patologia se caracteriza por tom grave acompanhado de turbulências aéreas em forma de ruído audível ${ }^{42}$.

Dentro do grupo de DOF, o TMF /z/ mostrou predomínio significativo da falta de coaptação glótica (Tabela 2), o que ocorreu também quando comparado ao grupo de DFUIV (Tabela 7 ), fato que está de acordo com a literatura que afirma que, quando uma lesão de prega vocal está presente, o TMF encontra-se reduzido. Essa inabilidade em sustentar a fonação se deve à aproximação insuficiente das pregas vocais devido à presença da lesão, com conseqüente incoordenação do esforço respiratório com o fechamento glótico ${ }^{13}$, ou até mesmo a redução do TMF /z/ pode representar uma estratégia compensatória mal adaptada ${ }^{26}$.

No grupo de DFUIV, o TMF /z/ mostrou percentuais indicativos de falta de coaptação glótica significativamente maiores do que os indicativos de hipercontração glótica (Tabela 2). Esse dado sugere que a tensão à fonação não foi predominante, ao contrário do que se esperava, uma vez que a hiperfunção vocal é uma das principais características das alterações vocais nas DFUIV ${ }^{27}$. Diante deste achado, supõe-se estar diante de um resultado de hipofunção secundária à hiperfunção fonatória, o que vai ao encontro da literatura que cita ser a fadiga neuromuscular uma redução da capacidade de um músculo sustentar determinada contração sob estimulação repetida. A fadiga da musculatura intrínseca e/ou extrínseca da laringe pode potencialmente resultar em capacidade reduzida para manter a tensão das pregas vocais e a estabilidade da postura laríngea ${ }^{21,37}$.

Constatou-se ocorrência significativa de DOF, o que sugere alta freqüência de sujeitos que vão à procura de tratamento fonoaudiológico para DFUIV tardiamente, ou seja, quando já apresentam lesão em nível de prega vocal.

Nas DOF e DFUIV, a maioria significativa dos pacientes, apesar de apresentarem a relação $\mathrm{s} / \mathrm{z}$ normal, apresentou tempos isolados de $/ \mathrm{s} / \mathrm{e} / \mathrm{z} /$ abaixo do normal, sugerindo dificuldades no fechamento glótico ou na manutenção do controle respiratório, podendo sobrecarregar a laringe, o que indica que o resultado da relação s/z não deve ser utilizado isoladamente para diagnóstico da disfonia e sim como um indicador, dentro de um contexto maior de avaliações.

Dentro da DOF, a falta de coaptação glótica teve percentuais consideráveis tanto na relação s/z quanto no TMF /z/ isoladamente, sugerindo presença de lesão em nível de prega vocal, o que é compatível com o fato da presença de alteração 
vocal ter sido estatisticamente significativa, com predomínio, também significativo, da voz ruidosa, sendo que os nódulos vocais foram significativamente mais freqüentes do que as demais patologias neste grupo. Esses dados sugerem que a presença de patologia em nível de prega vocal tem um papel deletério e geralmente ocasiona a alteração da qualidade perceptivo-auditiva da voz.

O tipo vocal alterado mais encontrado na DFUIV foi a voz ruidosa, consistindo num sinal de alerta para diagnóstico precoce de uma possível DOF.

\section{CONCLUSÃO}

O resultado da relação $s / z$ foi estatisticamente significativo a favor da faixa de normalidade estabelecida, tanto nos pacientes com diagnóstico de DOF, quanto naqueles com DFUIV, sendo que, em ambos os grupos de pacientes, a voz ruidosa foi a mais freqüente. Dentro das DOF, os nódulos vocais foram significativamente mais freqüentes do que as demais patologias.

\begin{abstract}
Purpose: to check the result of $s / z$ ratio and types of voice in patients with diagnosis of organicfunctional dysphonias (DOF) and functional dysphonias by incorrect use of voice (DFUIV), as well as the occurrence of the various pathologies within the DOF. Methods: 70 subjects of both genders, between 5 and 65 years old, seen in a clinic-school, registered in a data bank from 1998 to 2006, with DOF and DFUIV, both classified in three sub-groups: cases in which the s/z ratio indicated hypercontraction, normality and lack of coaptation of the vocal folds during phonation. According to the occurrence, the types of voice were classified in: without alteration; noisy; compressed and/or presenting alteration of $f_{0} h$; noisy and/or with alteration of $f_{0} h$; and compressed. Results: significant presence of DOF and DOF with vocal nodules; in DOF and DFUIV, significant presence of the normal $\mathrm{s} / \mathrm{z}$ ratio with isolated times of $/ \mathrm{s} /$ and $/ \mathrm{z} /$ below the normal and noisy voice. Conclusions: the result of the s/z ratio was statistically significant in favor of the range of established normalcy, both in patients with a diagnosis of DOF, as well as with those with DFUIV, and that, in both groups of patients, the noisy voice was the most frequent. Within the DOF, the vocal nodules were significantly more frequent than the other pathologies.
\end{abstract}

KEYWORDS: Voice; Phonation; Voice Disorders; Health Evaluation

\section{REFERÊNCIAS}

1. Fernández CAP, López JP. Nódulos de cuerdas vocales, factores de riesgo en los docentes: estudio de casos y controles. Acta Otorrinolaringol Esp. 2003; 54(4):253-60.

2. Behrman A, Sulica L, He T. Factors predicting patient perception of dysphonia caused by benign vocal fold lesions. Laryngoscope. 2004; 114(10):1693-700.

3. Demmink-Geertman L, Dejonckere PH. Nonorganic habitual dysphonia and autonomic dysfunction. J Voice. 2002; 16(4):549-59.

4. Jilek C, Marienhagen J, Hacki T. Vocal stability in functional dysphonic versus healthy voices at different times of voice loading. J Voice. 2004; 18(4):443-53.

5. Weigelt S, Krischke S, Klotz M, Hoppe U, Köllner $\mathrm{V}$, Eysholdt $\mathrm{U}$, et al. Voice handicap in patients with organic and functional dysphonia. Instrument zur Bestimmung der subjektiven Beeinträchtigung durch organische und funktionelle dysphonien. HNO. 2004; 52(8):751-6.

6. Kosztyla-Hojna B, Rogowski M, Ruczaj J, Pepinski W, Lobaczuk-Sitnik A. An analysis of occupational dysphonia diagnosed in the North-East of Poland. Int J Occup Med Environ Health. 2004; 17(2):273-8.

7. Souza OC, Hanayama EM. Fatores psicológicos associados a disfonia funcional e a nódulos vocais em adultos. Rev CEFAC. 2005; 7(3):388-97.

8. Pontes PAL, Vieira VP, Gonçalves MIR, Pontes AAL. Características das vozes roucas, ásperas e normais: análise acústica espectrográfica comparativa. Rev Bras Otorrinolaringol. 2002; 68(2):182-8.

9. Behlau M, Madazio G, Feijó D, Pontes P. Avaliação de voz. In: Behlau M. organizador. Voz: o livro do especialista. vol 1. Rio de Janeiro: Revinter; 2001. p. 85-172.

10. Awan SN, Roy N. Acoustic prediction of voice type in women with functional dysphonia. $\mathrm{J}$ Voice. 2005; 19(2):268-82. 
11. Oliveira IB. Avaliação fonoaudiológica da voz: reflexões sobre condutas, com enfoque à voz profissional. In: Ferreira LP, Befi-Lopes DM, Limongi SCO. Tratado de fonoaudiologia. São Paulo: Roca; 2004. p.11-25.

12. Mendes A, Castro E. Análise acústica da avaliação vocal I: tarefas fonatória e medidas acústicas. Rev Port Otorrinolaringol Cirur Cerv Fac. 2005; 43(2):127-36.

13. Colton RH, Casper JK. Compreendendo os problemas de voz: uma perspectiva fisiológica ao diagnóstico e ao tratamento. Porto Alegre: Artes Médicas; 1996. 386p.

14.Brasil.Lein.08.069, de13deJulhode1990.Estatuto da Criança e do Adolescente. Art. $2^{\circ}$. Disponível em: <http://www.estatutodacriancaedoadolescente. com/eca.htm>. Acesso em 30 set 2006.

15. Shigemori Y. Some tests related to the air usage during phonation. Clinical Investigations. Otologia. 1997; 23:138-66.

16. Tait NA, Michel JF, Carpenter MA. Maximum duration of sustained $/ \mathrm{s} /$ and $/ z /$ in children. J Speech Hear Disord. 1980; 45(2):239-46.

17. Finnegan DE. Maximum phonation time for children with normal voices. J Commun Disord. 1984; 17(5):309-17.

18. Prater RJ, Swift RN. Manual of voice teraphy. Boston: Little Brown; 1984. p. 10-24.

19. Eckel F, Boone D. The s/z ratio as an indicator of laryngeal pathology. J Speech Hear Disord. 1981; 46(2):147-56.

20. Gamboa FJ, Nieto A, del Palacio AJ, Rivera T, Cobeta I. [S/z ratio in glottic closure defects]. Acta Otorrinolaringol Esp. 1995; 46(1):45-8.

21. Pinho SMR. Avaliação e tratamento da voz. In: Pinho SMR. Fundamentos em fonoaudiologia: tratando os distúrbios de voz. 2. ed. Rio de Janeiro: Guanabara Koogan; 2003. p. 3-40.

22. Ferrone C, Leung G, Ramig LO. Fragments of a Greek Trilogy: impact on phonation. J Voice. 2004; 18(4):488-99.

23. Le Huche F, Allali A. A voz: patologia vocal de origem funcional. 2. ed. vol 2. Porto Alegre: Artmed; 2005.

24. Gelfer MP, Pazera JF. Maximum duration of sustained $/ \mathrm{s} /$ and $/ \mathrm{z} /$ and the $\mathrm{s} / \mathrm{z}$ ratio with controlled intensity. J Voice. 2006; 20(3):369-79.

25. Larson GW, Mueller PB, Summers PA. Effects of procedural variations in determining the $s / z$ ratio of young children. J Brit Coll Speech Therap. 1990; 863:7-8.

26. Steffen LM, Moschetti MB, Steffen N, Hanayama EM. Paralisia unilateral de prega vocal: associação e correlação entre tempos máximos de fonação, posição e ângulo de afastamento. Rev Bras Otorrinolaringol. 2004; 70(4):450-5.
27. Navas DM, Dias PR. Disfonias funcionais. In: Pinho SMR. Fundamentos em fonoaudiologia: tratando os distúrbios da voz. 2. ed. Rio de Janeiro: Guanabara Koogan; 2003. p. 77-81.

28. Melnyk $P$, Jamardo $B$, Cacace $M$, Pardo $\mathrm{H}$, Pino AA, Tomasetti $A$, et al. Considerations about teachers' dysphonias. Int Congr Ser. 2003; 1240:1293-6.

29. Andrade LMA. Determinação dos limiares de normalidade dos parâmetros acústicos da voz [dissertação]. São Paulo (SP): Universidade de São Paulo; 2003.

30. Master S, Biase ND, Chiari BM, Pedrosa V. $\mathrm{O}$ espectro médio de longo termo na pesquisa e na clínica fonoaudiológica. Pró-Fono. 2006; 18(1):111-20.

31. Johns MM. Update on the etiology, diagnosis and treatment of vocal fold nodules, polyps, and cysts. Curr Opin Otolaryngol Head Neck Surg. 2003; 11(6):456-61.

32. Braga JN, Oliveira DSF, Atherino CCT, Schott TCA, Silva JC. Nódulos vocais: análise anátomofuncional. Rev CEFAC. 2006; 8(2):223-9.

33. Behrman A, Dahl LD, Abramson AL, Schutte HK. Anterior-posterior and medial compression of the supraglottis: signs of nonorganic dysphonia or normal postures? J Voice. 2002; 17(3):403-10.

34. Paes C, Vieira J, Leonel T, Cunha DA. O impacto da respiração oral no comportamento vocal. J Bras Fonoaudiol. 2005; 5(23):417-23.

35. Silva MAA, Duprat AC. Voz cantada. In: Ferreira LP, Befi-Lopes DM, Limongi SCO. Tratado de fonoaudiologia. São Paulo: Roca; 2004. p.177-94.

36. Ortiz E, Costa EA, Spina AL, Crespo AN. Proposta de modelo de atendimento multidisciplinar para disfonias relacionadas ao trabalho: estudo preliminar. Rev Bras Otorrinolaringol. 2004; 70(5):590-6.

37. Welham NV, Maclagan MA. Vocal fatigue: currente knowledge and future directions. J Voice. 2003; 17(1):21-30.

38. Sataloff TS. Anatomia funcional e fisiologia da voz. In: Sataloff TS, Gould WJ, Spiegel JR. Manual prático de fonocirurgia. Rio de Janeiro: Revinter; 2002. p. 63-176.

39. Jotz GP, Cervantes O, Abrahão M, Settanni FAP, Angelis EC. Noise-to-harmonics ratio as an acoustic measure of voice disorders in boys. J Voice. 2002; 16(1):28-31.

40. Carding PN, Steen IN, Webb A, Mackenzie K, Deary IJ, Wilson JA. The reliability and sensitivity to change of acoustic measures of voice quality. Clin Otolaryngol All Scie. 2004; 29(5):538-44. 
41. Felippe ACN, Grillo MHMM, Grechi TH. Normatização de medidas acústicas para vozes normais. Rev Bras Otorrinolaringol. 2006; 72(5):659-64.
42. Batalla FN, Santos PC, Suárez Nieto C, Gonzáles BS, Sequeiros G. Evaluación perceptual de la disfonía: correlación con los parámetros acústicos y fiabilidad. Acta Otorrinolaringol Esp. 2004; 55(6):282-7.

RECEBIDO EM: 10/09/2007

ACEITO EM: 26/05/2008

Endereço para correspondência:

Rua Coronel Borges Fortes, 568 ap. 304

Santa Rosa - RS

CEP: $98900-000$

Tel: (55) 8404-1851

E-mail: gisifono@yahoo.com.br 